\title{
Body adiposity index, body fat content and incidence of type 2 diabetes
}

\author{
M. B. Schulze • B. Thorand • A. Fritsche • H. U. Häring • \\ F. Schick • A. Zierer • W. Rathmann • J. Kröger • \\ A. Peters $\cdot$ H. Boeing $\cdot$ N. Stefan
}

Received: 2 December 2011 / Accepted: 16 January 2012 /Published online: 17 February 2012

(C) Springer-Verlag 2012

\begin{abstract}
Aims/hypothesis The aim of this study was to compare estimates of body fat content, i.e. body adiposity index (BAI), BMI and waist and hip circumferences, with respect to their ability to predict the percentage of body fat (PBF; confirmed by magnetic resonance tomography) and incident type 2 diabetes.

Methods Associations between anthropometric measurements and PBF were evaluated in the Tübingen Lifestyle Intervention Program (TULIP; 138 men, 222 women), and between these measurements and incident type 2 diabetes in the European Prospective Investigation into Cancer and Nutrition (EPIC)-Potsdam study (9,729 men, 15,438 women) and the Cooperative Health Research in the Region of Augsburg (KORA) study (5,573 men, 5,628 women), using correlation and multivariate Cox regression analyses.
\end{abstract}

Electronic supplementary material The online version of this article (doi:10.1007/s00125-012-2499-z) contains peer-reviewed but unedited supplementary material, which is available to authorised users.

\footnotetext{
M. B. Schulze $(\varangle) \cdot J$. Kröger

Department of Molecular Epidemiology,

German Institute of Human Nutrition Potsdam-Rehbruecke,

Arthur-Scheunert-Allee 114-116,

14558 Nuthetal, Germany

e-mail: mschulze@dife.de

B. Thorand $\cdot$ A. Zierer $\cdot$ A. Peters

Institute of Epidemiology II, Helmholtz Zentrum München,

German Research Center for Environmental Health,

Neuherberg, Germany

A. Fritsche $\cdot$ H. U. Häring $•$ N. Stefan

Department of Internal Medicine, Division of Endocrinology,

Diabetology, Nephrology, Vascular Disease and Clinical

Chemistry, University of Tübingen,

Tübingen, Germany
}

Results BMI more strongly correlated with PBF (men: $r=$ 0.81 , women: $r=0.84$ ) than BAI ( $r=0.68$ and 0.81 , respectively), while waist circumference among men $(r=0.84)$ and hip circumference among women $(r=0.88)$ showed the strongest correlations. BAI overestimated PBF among men (mean difference $-3.0 \%$ ), and this error was dependent on the value of PBF. BAI was more weakly associated with diabetes risk (RRs for 1 SD, EPIC-Potsdam men: 1.62 [95\% CI 1.52, 1.72], women: 1.67 [95\% CI 1.55, 1.80]; KORA men: 1.62 [95\% CI 1.48, 1.78], women: 1.82 [95\% CI 1.65, 2.02]) compared with BMI (RRs, EPIC-Potsdam men: 1.95 [95\% CI 1.83, 2.09], women 1.88 [95\% CI 1.76, 2.02], KORA men 1.75 [95\% CI 1.62, 1.89], women 2.00 [95\% CI 1.81, 2.22]), while waist circumference showed the strongest associations (RRs: 2.17 [95\% CI 2.01, 2.35], 2.33 [95\% CI 2.15, 2.53], 1.81 [95\% CI 1.66, 1.96] and

F. Schick

Section of Experimental Radiology, University of Tübingen,

Tübingen, Germany

W. Rathmann

Institute of Biometrics and Epidemiology,

German Diabetes Center, Leibniz Center for Diabetes

Research at Heinrich Heine University Düsseldorf,

Düsseldorf, Germany

H. Boeing

Department of Epidemiology,

German Institute of Human Nutrition Potsdam-Rehbruecke,

Nuthetal, Germany 
2.29 [95\% CI 2.05, 2.57] for EPIC-Potsdam men and women and KORA men and women, respectively).

Conclusions/interpretation Waist circumference in men and hip circumference in women are better predictors of PBF than BAI and BMI. BAI was not as strong a predictor of diabetes as BMI, while waist circumference was the strongest predictor.

Keywords Adiposity Body adiposity index $\cdot$ Body mass index $\cdot$ Cohort studies $\cdot$ Diabetes mellitus, type 2 .

Magnetic resonance imaging · Proportional hazards models .

ROC curve - Waist circumference

$\begin{array}{ll}\text { Abbreviations } \\ \text { BAI } & \begin{array}{l}\text { Body adiposity index } \\ \text { Dual-energy X-ray absorptiometry }\end{array} \\ \text { EXIC } & \begin{array}{l}\text { European Prospective Investigation into } \\ \text { Cancer and Nutrition } \\ \text { Cooperative Health Research in the } \\ \text { Region of Augsburg }\end{array} \\ \text { KORA } & \begin{array}{l}\text { MONItoring of Trends and Determinants } \\ \text { in CArdiovascular Diseases }\end{array} \\ \text { MONICA } & \begin{array}{l}\text { Magnetic resonance tomography } \\ \text { Percentage of body fat }\end{array} \\ \text { MRT } & \begin{array}{l}\text { Receiver operating characteristic } \\ \text { PB̈bingen Lifestyle Intervention Program }\end{array}\end{array}$

\section{Introduction}

The BMI has been selected by Keys et al. [1] as the best measure of body fatness, after Adolphe Quetelet first proposed the index (originally referred to as the Quetelet index) in 1871, and suggested that it was the best statistical approach to reflect growth patterns in humans. Since that time, the BMI has been the most commonly applied clinical measure to characterise obesity in individuals. However, given that, at the same BMI, the relative composition of fat mass vs lean body mass depends on age, sex and ethnicity [2], the BMI has been criticised as frequently being an inaccurate measure of body fatness. Recently, a new index reflecting body composition, the Body Adiposity Index (BAI) was proposed [3]. It was derived from correlation and regression analysis of anthropometric measurements and percentage of body fat (PBF) measured by dual-energy X-ray absorptiometry (DXA) in samples of Mexican-American and black individuals. The BAI allows computation of PBF with the same formula for men and women using hip circumference and height, and thus does not require the application of complex and costly imaging methods or DXA. It may also be advantageous compared with other less costly field methods for the determination of
PBF, such as the measurement of skinfold thickness or bioelectric impedance analysis, which have been criticised as being inaccurate $[4,5]$.

A general and important question now is whether established and newly developed estimates of adiposity can precisely measure body fat content in men and women, who are known to have large differences in body fat content and body fat distribution. Bergman et al. [3], for example, observed quite a strong correlation between body height and PBF measured by DXA, the main argument for including height in the calculation of BAI. However, this correlation may largely be driven by sex differences in both height and PBF. Thus, BAI seems to capture relative fatness as well as the well-established difference in body composition between men and women [6]. In contrast, BMI is an index of body adiposity specifically intended to be uncorrelated with height, and thus also not to reflect sex differences in PBF. The fact that BMI, together with sex, can be used to predict PBF quite accurately has been shown previously [7]. In addition, BAI was not more strongly correlated with PBF (measured by DXA) than BMI in a sex-stratified analysis in a recent study among black and white Americans [8]. However, it remains largely unclear whether the estimation of body fat using BAI involves any sex-specific, systematic error. Furthermore, BAI has not previously been compared with hip and waist circumferences as sex-specific correlates of body fatness. It is also questionable whether the estimation of PBF is advantageous in predicting obesity related risk markers or endpoints, such as type 2 diabetes, compared with BMI or body circumferences. No clear advantage has been seen in comparing PBF from DXA with BMI [9], and data for BAI are, so far, lacking.

We have therefore aimed to compare BAI and established estimates of adiposity with respect to their ability to predict $\mathrm{PBF}$ estimated from magnetic resonance tomography (MRT) and their association with insulin sensitivity and incident type 2 diabetes among men and women.

\section{Methods}

Tübingen Lifestyle Intervention Program (TULIP) Data from 360 white Europeans from the southern part of Germany were included in the analyses. They participated in an ongoing study on the pathophysiology of type 2 diabetes [10]. Individuals were included in the study when they fulfilled at least one of the following criteria: a family history of type 2 diabetes, a BMI $>27 \mathrm{~kg} / \mathrm{m}^{2}$ or a previous diagnosis of impaired glucose tolerance or gestational diabetes. All participants had measurements of body fat distribution determined by MRT and insulin sensitivity from the oral glucose tolerance test [10]. They were considered 
healthy according to a physical examination and routine laboratory tests. Informed written consent was obtained from all participants and the local medical ethics committee approved the protocol.

\section{European Prospective Investigation into Cancer and Nutrition} (EPIC)-Potsdam study The EPIC-Potsdam study includes 27,548 participants: 16,644 women aged mainly $35-65$ years and 10,904 men aged mainly 40-65 years, from the general population of Potsdam, Germany, recruited between 1994 and 1998 [11]. Informed consent was obtained from all participants, and approval was given by the Ethical Committee of the State of Brandenburg, Germany. The baseline examination included anthropometric measurements, a personal interview and a questionnaire on prevalent diseases and sociodemographic and lifestyle characteristics, as well as a validated semi-quantitative food frequency questionnaire. The anthropometric measurement procedures and measures of quality control have been previously described in detail $[12,13]$. Briefly, body weights were measured using electronic digital scales, accurate to $100 \mathrm{~g}$, with participants wearing only light underwear and after emptying the bladder. Heights were measured to the nearest $0.1 \mathrm{~cm}$ using a flexible anthropometer. Waist circumference was measured midway between the lower rib margin and the superior anterior iliac spine. Hip circumference was taken at the widest point over the greater trochanters. Both were taken to the nearest $0.5 \mathrm{~cm}$ and measured with a nonstretching tape applied horizontally, with proper use controlled by a mirror.

Follow-up questionnaires have been administered every 2-3 years. Response rates for follow-up rounds have exceeded $90 \%$. Incident cases of diabetes were identified up to August 2005 via self-reports of a diabetes diagnosis, diabetes-relevant medication or dietary treatment because of diabetes. All cases were verified by the diagnosing physician using ICD-10 (www.who.int/classifications/icd/en/). After exclusion of participants with prevalent diabetes, with self-reported diabetes during follow-up but without confirmation by a physician, with missing follow-up time, and with missing covariate information at baseline, 9,729 men and 15,438 women remained for analyses.

Cooperative Health Research in the Region of Augsburg (KORA) The data were derived from the second, third and fourth population-based MONICA (MONItoring of Trends and Determinants in CArdiovascular Diseases)/KORA Augsburg (southern Germany) surveys (S2-S4) conducted in 1989/90 (S2), 1994/95 (S3) and 1999/2001 (S4) [14, 15]. A total of 13,871 individuals $(6,891$ men, 6,980 women; response: $67-77 \%$ ) aged $25-74$ years participated in $\geq 1$ of the three cross-sectional studies. All participants were prospectively followed within the framework of the KORA studies. The incidence of diabetes was assessed using follow-up questionnaires sent to the participants in 1997/ 98, 2002/03 and 2008/09 (S4 only) or personal interviews conducted in 2006/08 (S4 only). Cases with self-reported incident diabetes were validated by contacting the treating physician or by medical chart review and only participants for whom the treating physician clearly reported a diagnosis of type 2 diabetes, or for whom a diagnosis of type 2 diabetes was mentioned in the medical records, or who were taking glucose-lowering medication, were classified as having type 2 diabetes. We excluded all individuals with prevalent type 2 diabetes at baseline $(n=702)$ and with missing follow-up information concerning incident diabetes $(n=$ $1,765)$. Furthermore, participants with incomplete data for any of the covariables $(n=203)$ were excluded. Finally, the prospective analyses included 11,201 non-diabetic study participants (5,573 men, 5,628 women) aged 25-74 years at baseline. Written informed consent was obtained from each study participant, and the study was approved by the local authorities.

Baseline information on sociodemographic variables, physical activity level, and alcohol consumption was gathered by trained medical staff during a standardised interview. In addition, all participants underwent an extensive standardised medical examination that included the collection of a nonfasting blood sample. All measurement procedures have been described elsewhere in detail $[14,16]$. Anthropometric measurements were taken after the participants had removed their shoes, heavy clothing and belts. Body weight was measured to the nearest $0.1 \mathrm{~kg}$ and height to the nearest $0.5 \mathrm{~cm}$ while the participants were wearing light clothing. Waist circumference was measured at the level midway between the lower rib margin and the iliac crest while the participants breathed out gently. Hip circumference was taken at the level of maximal gluteal protrusion [17].

Statistical analysis BMI was calculated as body weight $(\mathrm{kg})$ divided by squared height $\left(\mathrm{m}^{2}\right)$, the BAI was calculated using hip circumference and height $(\mathrm{BAI}=[\mathrm{hip}(\mathrm{cm}) /$ height $(\mathrm{m})^{1.5}$ ] 18$)$ [3]. We used correlation analysis to compare BAI and other body fat measures with PBF, determined by MRT, in the TULIP study. To assess agreement between BAI and PBF from MRT and to detect any bias with the test method relative to the reference method, differences between the two respective methods were plotted against the mean, as suggested by Bland and Altman [18]. We estimated the RRs comparing quintiles of anthropometric characteristics, and for a $1 \mathrm{SD}$ difference using Cox proportional hazards analysis separated for men and women in the EPIC-Potsdam and KORA studies. Study participants were censored at the date of diagnosis of diabetes, death or last follow-up ascertainment. We used information on covariates obtained from the baseline examination in multivariate analyses, including 
Table 1 Pearson correlation between anthropometric characteristics and PBF from MRT and insulin sensitivity, TULIP study

\begin{tabular}{|c|c|c|c|c|c|c|}
\hline \multirow[t]{2}{*}{ Characteristic } & \multicolumn{3}{|l|}{ PBF (MRT) } & \multicolumn{3}{|l|}{ Insulin sensitivity } \\
\hline & All & Men & Women & All & Men & Women \\
\hline Age (years) & -0.002 & -0.13 & 0.13 & -0.16 & -0.30 & -0.08 \\
\hline Height $(\mathrm{cm})$ & -0.40 & 0.14 & -0.09 & 0.13 & 0.10 & 0.14 \\
\hline Weight (kg) & 0.35 & 0.77 & 0.79 & -0.51 & -0.53 & -0.54 \\
\hline Waist circumference $(\mathrm{cm})$ & $0.34(p<0.001)$ & $0.84(p<0.001)$ & $0.76(p=0.041)$ & $-0.57(p=0.16)$ & $-0.61(p=0.082)$ & $-0.59(p=0.15)$ \\
\hline Hip circumference (cm) & $0.76(p<0.001)$ & $0.77(p=0.007)$ & $0.88(p<0.001)$ & $-0.52(p=0.47)$ & $-0.49(p=0.65)$ & $-0.53(p=0.71)$ \\
\hline BMI $\left(\mathrm{kg} / \mathrm{m}^{2}\right)$ & $0.65(p<0.001)$ & $0.81(p<0.001)$ & $0.84(p=0.087)$ & $-0.57(p=0.044)$ & $-0.57(p=0.21)$ & $-0.57(p=0.27)$ \\
\hline BAI (body fat, \%) & 0.83 & 0.68 & 0.81 & -0.50 & -0.51 & -0.54 \\
\hline
\end{tabular}

$p$ values are given for comparisons of correlation coefficients with those observed for BAI

age, education, smoking, alcohol consumption and physical activity. We compared the discriminative power of anthropometric characteristics on risk of type 2 diabetes through receiver operating characteristic (ROC) curve analysis. Analyses were performed using SAS release 9.2 (SAS Institute, Cary, NC, USA) and JMP 5.1. All $p$ values are two-tailed; $p<0.05$ was considered statistically significant.

\section{Results}

Baseline characteristics of the three study populations are presented in the electronic supplementary material [ESM] Table 1. Participants in the TULIP study were on average slightly younger (men: 46 years, women: 45 years) and had larger mean BMI and waist and hip circumferences compared with participants of EPIC-Potsdam (52 and 48 years) and KORA (49 and 48 years).

Table 1 shows the correlation between anthropometric variables and both PBF determined by MRT and insulin sensitivity in the TULIP study. Height was inversely associated with PBF in the whole study population $(r=-0.40)$. Hip circumference was considerably more strongly correlated with PBF than waist circumference. In addition, BMI was more weakly correlated with PBF than BAI ( $r=0.65$ vs $0.83, p<0.001)$. Correlations were considerably different if men and women were investigated separately. While hip circumference was most strongly correlated with PBF among women $(r=0.88)$, waist circumference showed the strongest correlation of all anthropometric variables among men $(r=0.84)$. BMI was more strongly correlated when sexes were analysed separately. Particularly among men, BAI appeared not to be a good measure of PBF $(r=0.68)$ compared with BMI $(r=0.81 ; p<0.001)$. Figure 1 shows Bland and Altman's limits-of-agreement plots between PBF estimated by BAI and MRT. BAI overestimates PBF among men (mean difference: $-3.0 \%$ ) and only slightly underestimates PBF among women $(0.4 \%)$. This error is dependent on the absolute amount of body fat, particularly among men. No large differences between men and women were apparent with regard to correlations of anthropometric
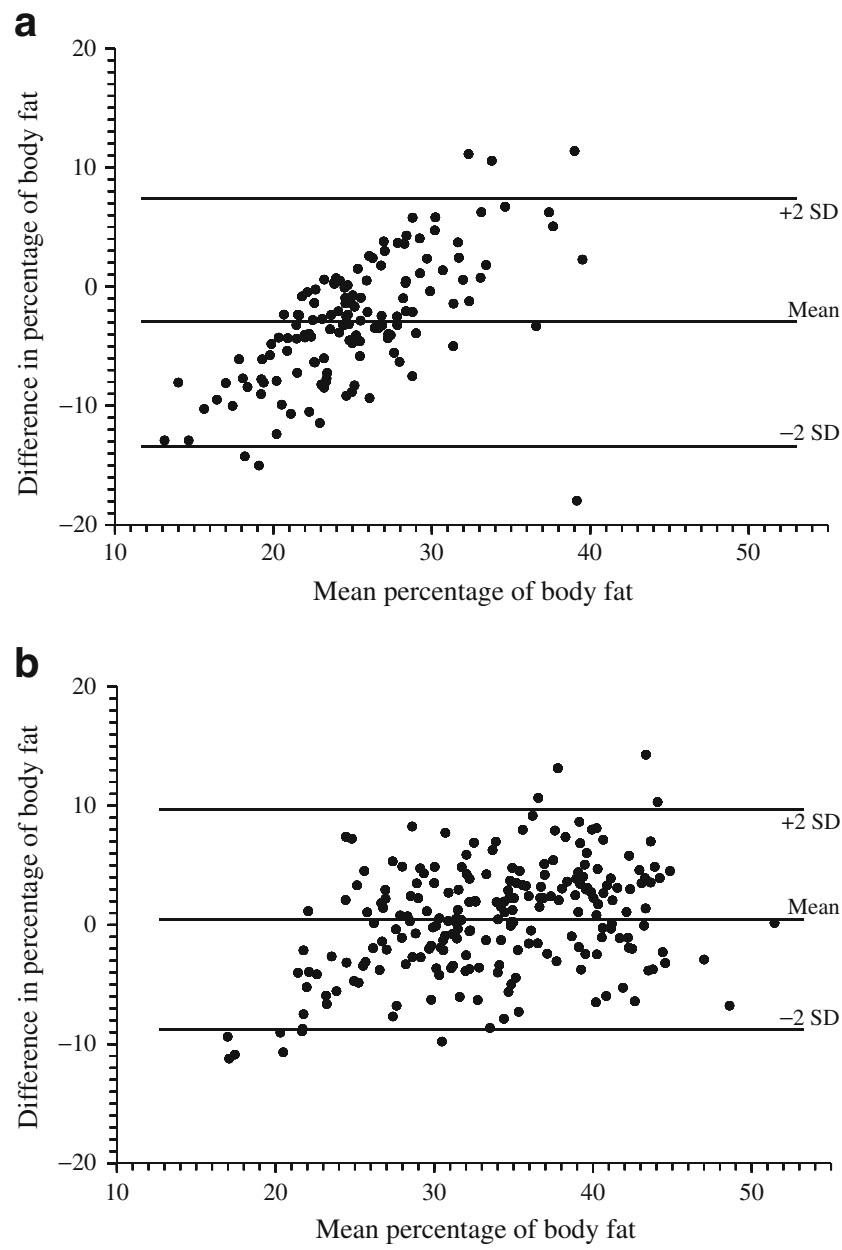

Fig. 1 Difference against mean for PBF, estimated by MRT and BAI, among men (a) and women (b) in the TULIP study. The difference between both methods for estimating PBF (MRT and BAI) is plotted against the mean from the two methods, with mean difference and mean \pm 2 SD marked 
characteristics and insulin sensitivity (Table 1$)$. BAI $(r=-0.50)$ was slightly more weakly associated with insulin sensitivity than BMI $(r=-0.57, p=0.044)$. Waist circumference showed the strongest correlation with insulin sensitivity among men and women, although correlations were not statistically significantly different from those for BAI.

During the 7 years of follow-up we observed 849 incident cases of type 2 diabetes (492 men, 357 women) in EPIC-Potsdam. In the KORA study, 574 incident cases of type 2 diabetes (340 men, 234 women) were observed during a mean follow-up time of 9 years. All anthropometric measurements were significantly positively associated with diabetes risk in men and women independent of age, and adjustment for other diabetes risk factors had only a small impact on these associations (Table 2). The strongest associations among men were observed for waist circumference (RR for 1 SD: 2.17 [95\% CI 2.01, 2.35] in EPIC-Potsdam, $1.81[95 \%$ CI $1.66,1.96]$ in KORA) followed by BMI (RR for 1 SD: 1.95 [95\% CI 1.83, 2.09] in EPIC-Potsdam, 1.75 [95\% CI 1.62, 1.89] in KORA). BAI was more weakly associated (RR for $1 \mathrm{SD}: 1.62$ [95\% CI 1.52, 1.72] in EPIC-Potsdam, 1.62 [95\% CI 1.48, 1.78] in KORA), relatively similar to hip circumference (RR for $1 \mathrm{SD}: 1.58$ [95\% CI 1.48, 1.68] in EPIC-Potsdam, 1.47 [95\% CI 1.36, 1.58] in KORA). Waist circumference showed the strongest association among women (RR for 1 SD: 2.33 [95\% CI 2.15, 2.53] in EPIC-Potsdam, 2.29 [95\% CI 2.05, 2.57] in KORA). The strength of association was weaker for BMI (RR for 1 SD: 1.88 [95\% CI 1.76, 2.02] in EPIC-Potsdam, 2.00 [95\% CI 1.81, 2.22] in KORA), BAI (RRs: 1.67 [95\%
CI $1.55,1.80$ ] and 1.82 [95\% CI 1.65, 2.02], respectively), and hip circumference (RRs 1.67 [95\% CI 1.56, 1.80] and 1.63 [95\% CI 1.49, 1.78], respectively).

Evaluating quintiles, we recorded nearly log-linear associations of anthropometric measurements with diabetes risk (Fig. 2). Among men, BMI (RRs comparing extreme quintiles: 14.8 [95\% CI 8.91, 24.7] in EPIC-Potsdam, 7.95 [95\% CI $4.65,13.6]$ in KORA) and waist circumference (RRs: 11.6 [95\% CI 7.24, 18.6] and 6.78 [95\% CI 4.05, 11.4], respectively) were stronger related to diabetes risk compared with BAI (RRs: 7.76 [95\% CI 5.09, 11.8] and 4.81 [95\% CI 2.87, 8.03], respectively) and hip circumference (RRs: 5.40 [95\% CI 3.80, 7.67] and 3.49 [95\% CI 2.38, 5.13], respectively). Among women, the strength of association was relatively similar for BMI, BAI and hip circumference in EPIC-Potsdam (RRs comparing extreme quintiles: 11.9 [95\% CI 6.45, 22.1], 12.9 [95\% CI 6.31, 26.5] and 9.85 [95\% CI 5.58, 17.4], respectively), but considerably weaker than for waist circumference (RR: 24.8 [95\% CI 10.9, 56.2]). Associations were relatively similar for BAI, BMI and waist circumference for women in the KORA study (RRs comparing upper quintile with lower two quintiles: $16.6[95 \% \mathrm{CI}$ 8.43, 32.5], 15.0 [95\% CI 8.16, 27.5] and 13.1 [95\% CI 7.51, 23.0], respectively), while hip circumference showed a considerably weaker association with risk (RR: 6.56 [95\% CI $4.20,10.3])$.

ROC analyses revealed that, compared with models using BAI, models including BMI or waist circumference had higher discrimination accuracy, while models with hip circumference showed similar performance (Table 2, ESM Fig. 1).

Table 2 Anthropometric characteristics and incidence of type 2 diabetes, EPIC-Potsdam study and KORA S2-S4 studies

\begin{tabular}{|c|c|c|c|c|c|c|c|c|}
\hline \multirow[t]{2}{*}{ Characteristic } & \multicolumn{2}{|l|}{ BAI (body fat, \%) } & \multicolumn{2}{|l|}{ BMI $\left(\mathrm{kg} / \mathrm{m}^{2}\right)$} & \multicolumn{2}{|c|}{ Waist circumference $(\mathrm{cm})$} & \multicolumn{2}{|c|}{ Hip circumference $(\mathrm{cm})$} \\
\hline & $\operatorname{RR}(95 \% \mathrm{CI})^{\mathrm{a}}$ & $\begin{array}{l}\text { ROC- } \\
\text { AUC }^{\mathrm{b}}\end{array}$ & $\mathrm{RR}(95 \% \mathrm{CI})$ & $\begin{array}{l}\text { ROC- } \\
\text { AUC }\end{array}$ & $\mathrm{RR}(95 \% \mathrm{CI})$ & $\begin{array}{l}\text { ROC- } \\
\text { AUC }\end{array}$ & $\mathrm{RR}(95 \% \mathrm{CI})$ & $\begin{array}{l}\text { ROC- } \\
\text { AUC }\end{array}$ \\
\hline \multicolumn{9}{|l|}{ EPIC-Potsdam men } \\
\hline Age-adjusted & $1.68(1.58,1.79)$ & 0.726 & $2.03(1.91,2.17)$ & 0.770 & $2.27(2.11,2.45)$ & 0.770 & $1.64(1.54,1.74)$ & 0.715 \\
\hline Multivariate-adjusted $^{\mathrm{c}}$ & $1.62(1.52,1.72)$ & 0.752 & $1.95(1.83,2.09)$ & 0.787 & $2.17(2.01,2.35)$ & 0.784 & $1.58(1.48,1.68)$ & 0.746 \\
\hline \multicolumn{9}{|l|}{ EPIC-Potsdam women } \\
\hline Age-adjusted & $1.74(1.62,1.87)$ & 0.786 & $1.95(1.82,2.08)$ & 0.822 & $2.42(2.24,2.62)$ & 0.844 & $1.75(1.64,1.88)$ & 0.788 \\
\hline Multivariate-adjusted & $1.67(1.55,1.80)$ & 0.797 & $1.88(1.76,2.02)$ & 0.830 & $2.33(2.15,2.53)$ & 0.851 & $1.67(1.56,1.80)$ & 0.802 \\
\hline \multicolumn{9}{|l|}{ KORA men } \\
\hline Age and survey-adjusted & $1.63(1.49,1.78)$ & 0.757 & $1.74(1.61,1.88)$ & 0.772 & $1.81(1.67,1.96)$ & 0.774 & $1.45(1.35,1.57)$ & 0.749 \\
\hline Multivariate-adjusted & $1.62(1.48,1.78)$ & 0.772 & $1.75(1.62,1.89)$ & 0.787 & $1.81(1.66,1.96)$ & 0.788 & $1.47(1.36,1.58)$ & 0.768 \\
\hline \multicolumn{9}{|l|}{ KORA women } \\
\hline Age and survey-adjusted & $1.89(1.72,2.09)$ & 0.781 & $2.05(1.87,2.26)$ & 0.802 & $2.36(2.12,2.63)$ & 0.817 & $1.70(1.56,1.86)$ & 0.772 \\
\hline Multivariate-adjusted & $1.82(1.65,2.02)$ & 0.787 & $2.00(1.81,2.22)$ & 0.807 & $2.29(2.05,2.57)$ & 0.821 & $1.63(1.49,1.78)$ & 0.779 \\
\hline
\end{tabular}

${ }^{\mathrm{a}} \mathrm{RRs}$ were estimated for a $1 \mathrm{SD}$ difference

${ }^{\mathrm{b}} \mathrm{AUC}$ of the ROC for the specified model with anthropometric measures modelled as continuous variables

${ }^{\mathrm{c}}$ Adjusted for age, survey (KORA only), education, smoking, alcohol consumption and physical activity 
Fig. 2 Risk of type 2 diabetes for quintiles of BAI, BMI, waist circumference and hip circumference among men (a-d) and women $(\mathbf{e}-\mathbf{h})$ in the EPIC-Potsdam study (black squares) and KORA study (white circles) $a$
0
0
0
0
0
$\frac{0}{2}$

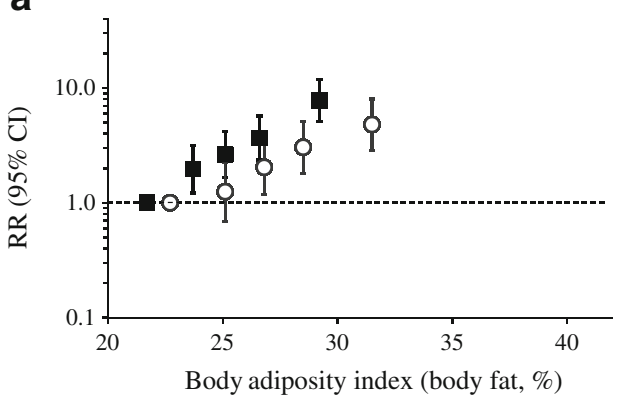

b

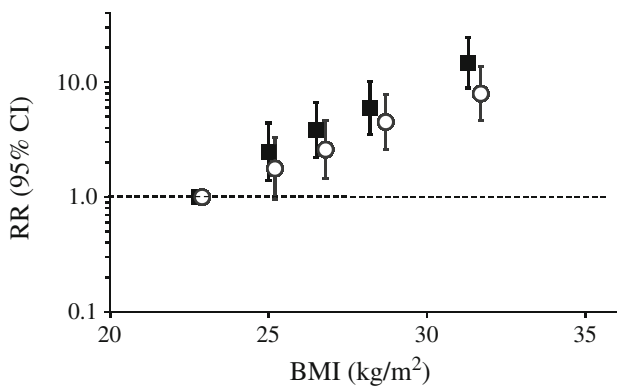

C

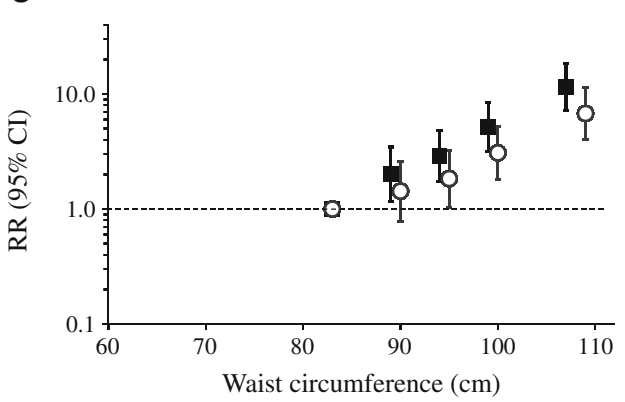

d

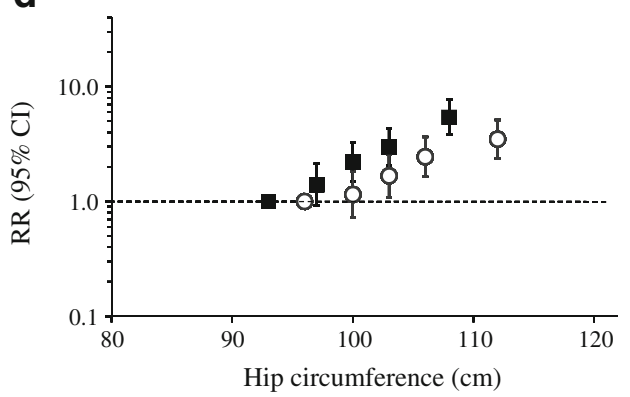

e

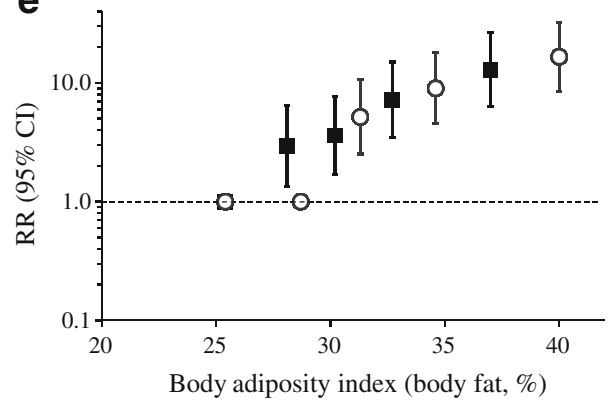

f

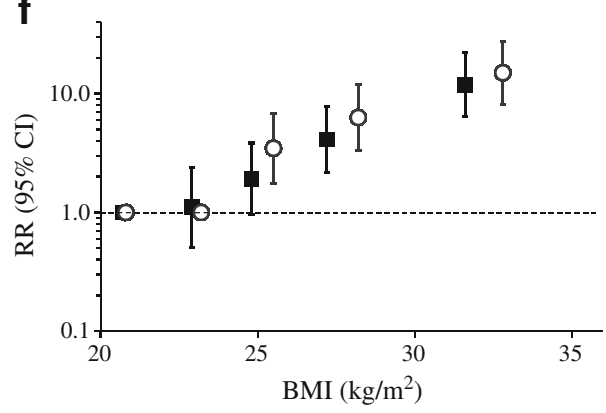

g

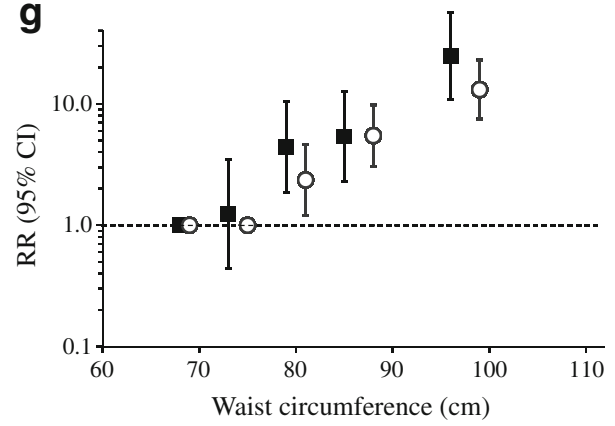

h

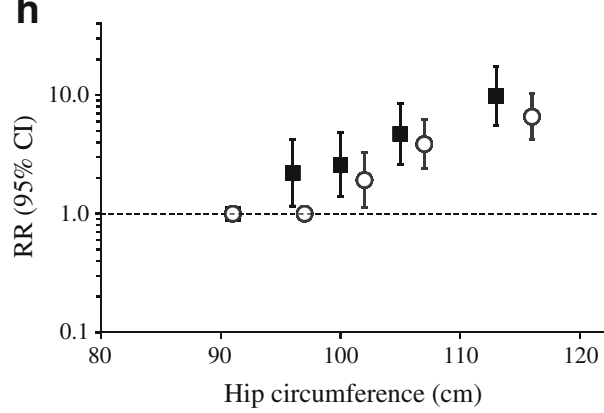

\section{Discussion}

By using the MRT technique we addressed the first aim of the study: to compare estimates of body fat content, i.e. BAI, BMI, and waist and hip circumferences with respect to their ability to predict PBF. Over recent years, MRT has proved to be a reliable tool for the very accurate assessment of total body fat content and body fat distribution. Simple T1-weighted techniques enable valid differentiation between lean tissue (mainly muscle and organs) and adipose tissue, owing to the different longitudinal relaxation times of protons bound to water and fat. We observed stronger correlations of waist circumference among men and hip circumference among women with PBF compared with BAI. The BAI was not a better predictor of PBF compared with BMI when considering men and women separately. This appears to be in contrast to the observation by Bergman et al. [3], who reported that BAI was considerably better correlated with PBF than BMI. However, this observation seems largely to have been driven by capturing sex differences in height and PBF when evaluating BAI in a mixed sample of men and women. Height was neither in men nor 
women a predominant determinant of PBF in our study. Our findings corroborate those from a recent study comparing BAI and BMI with PBF determined by DXA among black and white Americans [8]. Here, BAI was more strongly correlated with PBF than BMI when data from men and women were pooled. However, BAI was not superior compared with BMI in reflecting PBF in sex-stratified analysis.

Our data also indicate that waist circumference is by far the better indicator of PBF among men compared with hip circumference. Calculation of BAI would, therefore, result in unnecessarily inaccurate estimates of PBF among men. If body circumferences are to be implemented to better reflect PBF in men and women, there likely needs to be a sexspecific approach with measurements of hip circumference among women and waist circumference among men. Whether this is indeed an improvement to the use of BMI appears uncertain - waist and hip circumference were only slightly more strongly correlated with PBF than BMI. In addition, while waist circumference is not only a measure of general body fatness but also recommended for clinical practice as a measure of abdominal or central adiposity [19], the biological interpretation of hip circumference is less straightforward as it may reflect the accumulation of subcutaneous fat, greater gluteal muscle mass, or larger bone structure.

We then addressed the second aim of the study: to investigate the ability of estimates of body fat content to predict incident type 2 diabetes. BAI showed considerably weaker associations with diabetes risk than did BMI and waist circumference among men. Among women, associations with diabetes risk were relatively similar for BAI and BMI, but considerably weaker compared with waist circumference in one of the two cohorts. ROC analyses showed generally superior discrimination with BMI or waist circumference compared with BAI.

Whether the determination of PBF has advantages for the determination of metabolic risk factors of chronic disease risk compared with the classical determination of BMI or the measurement of circumferences has not clearly been shown so far. In a representative US population, BMI and waist circumference were correlated similarly well with biological risk markers compared with DXA measurements [9]. Similarly, objectively measured PBF from DXA [20-22], MRI measurements [23] or air-displacement plethysmography [24] was not more strongly related to cardiovascular risk factors than BMI or waist circumference in other studies. Our data suggest that an even more imprecise determination of PBF by calculating BAI based on measurements of hip circumference and height does not provide a meaningful alternative to BMI.

In a meta-analysis of prospective studies that evaluated BMI and waist circumference simultaneously, a 1 SD difference in BMI was slightly more weakly associated with risk (RR: 1.72 [95\% CI 1.47, 2.02]) than waist circumference
(RR: 1.87 [95\% CI 1.58, 2.20]) [25], which is corroborated by our findings. This suggests that anthropometric measurements that capture abdominal adiposity are alternatives to BMI in assessing type 2 diabetes risk in clinical practice and public health surveillance. However, both waist and hip circumference measurements involve difficulties in defining the measurement site and involve greater measurement error than the estimation of body weight and height $[12,26]$.

While the TULIP study population used to evaluate estimates of body fat content with respect to their ability to predict PBF from MRT was larger than the initial validation cohort (Triglyceride and Cardiovascular Risk in African-Americans study), it was considerably smaller than the BetaGene study used to derive the BAI [3] and another subsequent validation among White- and African-Americans [8]. Further subgroup analyses, for example by overweight/obesity status, were therefore not possible in TULIP. In addition, the TULIP study mainly included high-risk individuals, limiting the generalisability of our findings.

In conclusion, the BAI is not a better predictor than BMI of PBF determined by MRT. Waist and hip circumferences are determinants of PBF in a sex-dependent manner. In addition, BAI was not more strongly associated with diabetes incidence than BMI, and waist circumference was the strongest predictor in two large prospective studies in both sexes.

Funding The study was supported in part by a grant from the German Federal Ministry of Education and Research (BMBF) to the German Center for Diabetes Research (DZD e.V.). The TULIP study was in part supported by the Deutsche Forschungsgemeinschaft (KFO 114). N.S. is currently supported by a Heisenberg-Professorship from the Deutsche Forschungsgemeinschaft (STE 1096/3-1). The recruitment phase of the EPIC-Potsdam study was supported by the Federal Ministry of Science, Germany (01 EA 9401) and the European Union (SOC 9520140805 F02). The follow-up of the EPIC-Potsdam study was supported by German Cancer Aid (70-2488-Ha I) and the European Community (SOC 9820076905 F02). The KORA research platform (KORA, Cooperative Research in the Region of Augsburg) and the MONICA Augsburg studies were initiated and financed by the Helmholtz Zentrum München-German Research Center for Environmental Health, which is funded by the German Federal Ministry of Education and Research and by the State of Bavaria.

The sponsors of the study had no role in study design, data collection, data analysis, data interpretation, or writing of the report.

Duality of interest The authors declare that there is no duality of interest associated with this manuscript.

Contribution statement MBS analysed data and wrote the manuscript. BT and NS analysed data and revised the manuscript. AF, HUH, FS, AZ, WR, JK, AP and HB contributed to the interpretation of data and revised the manuscript. MBS, BT and NS had full access to the data in the study and take responsibility for the integrity of the data and the accuracy of the data analysis. All authors approved the final version. 


\section{References}

1. Keys A, Fidanza F, Karvonen MJ, Kimura N, Taylor HL (1972) Indices of relative weight and obesity. J Chronic Dis 25:329-343

2. Garn SM, Leonard WR, Hawthorne VM (1986) Three limitations of the body mass index. Am J Clin Nutr 44:996-997

3. Bergman RN, Stefanovski D, Buchanan TA et al (2011) A better index of body adiposity. Obesity (Silver Spring) 19:1083-1089

4. Marks GC, Habicht JP, Mueller WH (1989) Reliability, dependability, and precision of anthropometric measurements. The Second National Health and Nutrition Examination Survey 1976-1980. Am J Epidemiol 130:578-587

5. Sun G, French CR, Martin GR et al (2005) Comparison of multifrequency bioelectrical impedance analysis with dual-energy X-ray absorptiometry for assessment of percentage body fat in a large, healthy population. Am J Clin Nutr 81:74-78

6. Li C, Ford ES, Zhao G, Balluz LS, Giles WH (2009) Estimates of body composition with dual-energy $\mathrm{X}$-ray absorptiometry in adults. Am J Clin Nutr 90:1457-1465

7. Deurenberg P, Weststrate JA, Seidell JC (1991) Body mass index as a measure of body fatness: age- and sex-specific prediction formulas. Br J Nutr 65:105-114

8. Barreira TV, Harrington DM, Staiano AE, Heymsfield SB, Katzmarzyk PT (2011) Body adiposity index, body mass index, and body fat in white and black adults. JAMA 306:828-830

9. Sun Q, van Dam RM, Spiegelman D, Heymsfield SB, Willett WC, $\mathrm{Hu}$ FB (2010) Comparison of dual-energy X-ray absorptiometric and anthropometric measures of adiposity in relation to adiposityrelated biologic factors. Am J Epidemiol 172:1442-1454

10. Stefan N, Kantartzis K, Machann J et al (2008) Identification and characterization of metabolically benign obesity in humans. Arch Intern Med 168:1609-1616

11. Boeing H, Wahrendorf J, Becker N (1999) EPIC Germany-A source for studies into diet and risk of chronic diseases. Ann Nutr Metab 43:195-204

12. Klipstein-Grobusch K, Georg T, Boeing H (1997) Interviewer variability in anthropometric measurements and estimates of body composition. Int J Epidemiol 26(Suppl 1):S174-S180

13. Kroke A, Bergmann MM, Lotze G, Jeckel A, Klipstein-Grobusch K, Boeing H (1999) Measures of quality control in the German component of the EPIC study. European Prospective Investigation into Cancer and Nutrition. Ann Nutr Metab 43:216-224

14. Hense HW, Filipiak B, Döring A, Stieber J, Liese A, Keil U (1998) Ten-year trends of cardiovascular risk factors in the MONICA
Augsburg region in Southern Germany. Results from the 1984/ 85, 1989/90 and 1994/95 surveys. CVD Prev 1:318-327

15. Lowel H, Doring A, Schneider A, Heier M, Thorand B, Meisinger C (2005) The MONICA Augsburg surveys - basis for prospective cohort studies. Gesundheitswesen 67(Suppl 1):S13-S18

16. Meisinger C, Thorand B, Schneider A, Stieber J, Doring A, Lowel $\mathrm{H}$ (2002) Sex differences in risk factors for incident type 2 diabetes mellitus: the MONICA Augsburg cohort study. Arch Intern Med 162:82-89

17. Liese AD, Doring A, Hense HW, Keil U (2001) Five year changes in waist circumference, body mass index and obesity in Augsburg, Germany. Eur J Nutr 40:282-288

18. Bland JM, Altman DG (1986) Statistical methods for assessing agreement between two methods of clinical measurement. Lancet $1: 307-310$

19. Ness-Abramof R, Apovian CM (2008) Waist circumference measurement in clinical practice. Nutr Clin Pract 23:397-404

20. Lee K, Song YM, Sung J (2008) Which obesity indicators are better predictors of metabolic risk?: Healthy twin study. Obesity (Silver Spring) 16:834-840

21. Shen W, Punyanitya M, Chen J et al (2006) Waist circumference correlates with metabolic syndrome indicators better than percentage fat. Obesity (Silver Spring) 14:727-736

22. Steinberger J, Jacobs DR, Raatz S, Moran A, Hong CP, Sinaiko AR (2005) Comparison of body fatness measurements by BMI and skinfolds vs dual energy X-ray absorptiometry and their relation to cardiovascular risk factors in adolescents. Int $\mathrm{J}$ Obes (Lond) 29:1346-1352

23. Scherzer R, Shen W, Bacchetti P et al (2008) Simple anthropometric measures correlate with metabolic risk indicators as strongly as magnetic resonance imaging-measured adipose tissue depots in both HIV-infected and control subjects. Am J Clin Nutr 87:1809 1817

24. Bosy-Westphal A, Geisler C, Onur S et al (2006) Value of body fat mass vs anthropometric obesity indices in the assessment of metabolic risk factors. Int J Obes (Lond) 30:475-483

25. Vazquez G, Duval S, Jacobs DR Jr, Silventoinen K (2007) Comparison of body mass index, waist circumference, and waist/hip ratio in predicting incident diabetes: a meta-analysis. Epidemiol Rev 29:115-128

26. Nordhamn K, Sodergren E, Olsson E, Karlstrom B, Vessby B, Berglund L (2000) Reliability of anthropometric measurements in overweight and lean subjects: consequences for correlations between anthropometric and other variables. Int $\mathrm{J}$ Obes Relat Metab Disord 24:652-657 